\title{
Prediction of Depression Using Machine Learning and NLP Approach
}

\author{
Amrat Mali ${ }^{a}$, Dr. RR Sedamkar ${ }^{b}$ \\ Thakur College of Engineering and Technology, Mumbai a, Thakur College of Engineering and Technology, \\ Mumbai ${ }^{\text {, }}$ \\ amrat014@gmail.com ${ }^{\mathrm{a}}$,rr.sedamkar@thakureducation.org
}

\begin{abstract}
Today, for Internet users, micro-blogging has become a popular networking forum. Millions of people exchange views on different aspects of their lives. Thus, microblogging websites are a rich source of opinion mining data or Sentiment Analysis (SA) information. Because of the recent advent of microblogging, there are a few research papers dedicated to this subject. In our paper, we concentrate on Reddit.com, one of the leading microblogging sites, to explore the opinion of the public. We will demonstrate how to collect real-time Reddit data and use algorithms such as Support Vector Machine, KNN, and Multinomial Naive Bayes (MNB) for sentiment analysis or opinion mining purposes. We are able to assess positive and negative feelings using the algorithms selected above for the real-time Reddit depression info. The following experimental evaluations show that the algorithms used are accurate and can be used as an application for diagnosing the depression of individuals. After deployment User can write the content as input and after submitting the input text model API will be called from where result will come as User is going through depression or suicide. We worked with English in this post, but it can be used with any other language. English in this document, but it can be used for any other language this will be in future scope.
\end{abstract}

Keywords: NLP (Natural language Processing), Machine Learning, Reddit, Social networks, depression

\section{Introduction}

Depression as a common mental health disorder has long been defined as a single disease with a set of diagnostic criteria. It often co-occurs with anxiety or other psy-chological and physical disorders, and has an impact on feelings and behaviour of the affected individuals [1]. According to the WHO report, there are 322 million people expected to suffer from depression, equal to 4.4 percent of the global popula-tion. Nearly half of the in-risk individuals live in the South-East Asia (27 percent) and Western Pacific region (27 percent) like China and India. In many countries depression is still under-diagnosed and left without any adequate treatment which can lead into a serious self-perception and at its worst, to suicide [2]. In addition, the social stigma surrounding depression prevents many affected individuals from seek-ing an appropriate professional assistance. As a result, they turn to less formal re-sources such as social media. With the development of Internet usage, people have s-tarted to share their experiences and challenges with mental health disorders through online forums, micro-blogs or tweets.

Their online activities inspired many researchers to introduce new forms of potential health care solutions and methods for early depression detection systems. They tried to achieve higher performance improvements using various Natural Language Pro-cessing (NLP) techniques and text classification approaches. Some studies use single set features, such as bag of words (BOW) [3], [4], N-grams [5], LIWC [6] or LDA [7], [8] to identify depression in their posts. Some other papers compare the perfor-mance of individual features with various machine learning classifiers [9] - [12]

Recent studies examine the power of single features and their combinations such as N-grams + LIWC [13] or BOW+LDA and TF-IDF+LDA [14] to improve the accura-cy results. With almost 326 million active users and 90 million publicly distributed tweets to a wide audience, Twitter is one of the most popular social networking sites [14]. Many researchers have used Twitter data successfully as a source 
of insights into the epidemiology of users tweeting emotions, depression and other mental dis-orders.

As an online discussion site conducted by multiple groups or "sub-reddits," Reddit social media is widely used. It is also used for discussions on stigmatic subjects because it enables the users to be totally anonymous. The posts of Reddit users who wrote about mental health discourse were studied by Choudhury. Features such as self-concern, weak linguistic style, decreased social participation, and the expression of hopelessness or anxiety predicted this change.

\section{Related Work}

To provide new insight into depression detection, there are different types of re-search exploring the relationship between mental wellbeing and language use. Sig-mund Freud [13], dating back to the earliest years of psychology, wrote about Freud-ian slips or linguistic errors to expose the authors' inner thoughts and feelings. Vari-ous approaches to the relationship between depression and its language have been established through the development of sociology and psycholinguistic theories.

For example, according to the cognitive theory of depression of Aaron. [ 13], affect-ed people appear to view themselves and their environment in mostly negative terms. Via derogatory words and first-person pronouns, they also express them-selves. identify self-preoccupation as their typical feature, which can evolve into an intense stage of self-criticism. Other scholars have been inspired by these hypothe-ses to come up with empirical evidence for their validity.

For instance, in three separate periods of their lives, Stirman and Pennebaker [13] compared the word use of 300 poems written by 9 suicidal and 9 non-suicidal au-thors. The findings indicate that more first-person singular pronouns (I, me or we) were used by suicidal poets. Depressed students used more negative words and fewer positive words of feeling, according to his findings. In order to predict the improve-ment of depressive symptoms, Zinken, investigated the psychological im-portance of syntactic structures.

He assumed that in its word use, a written text may barely differ; however, it could differ in its syntactic structure, especially in the construction of relationships be-tween events. Analyzing the roles of cause and insight. Studies on depression and other mental health problems have brought new challenges with the advent of social media and the Internet era. In order to capture user behavioural patterns, online do-mains such as Facebook, Twitter or Reddit have provided a new forum for ground-breaking analysis with a rich source of text data and social metadata.

As an online discussion site conducted by various groups or "subreddits," Reddit social media is widely used. Since it allows the complete privacy of users, it is also used to address stigmatic subjects. Choudhury. Reviewed the posts of Reddit users who wrote about the debate on mental health and later moved to fix suicidal ideation problems. In the recent past, in a large research community, shared tasks potentially applicable to different circumstances have become significantly com-mon.

RISK, or the Early Risk Prediction Conference and Labs Assessment Forum, is a public competition that enables researchers from various disciplines to participate and collaborate on the production of 
reusable benchmarks for the assessment of early risk detection technologies used in various fields.

\section{Problem Statement}

Patients of mental problems such as Alzheimer's, depression, anxiety and neuro-degenerative diseases. The most depressed place in the world is India. Using Natural, after taking input as text data, results will be extracted from the dataset. Language processing algorithm and classification algorithm algorithm to find the data as de-pression data and prediction or not as a suicidal post.

\section{Proposed System}

Data Pre-Processing: We will take our first look at it now that we have received our data, looking for missing values and selecting which sections of the data set will be useful for our classifier. We're also going to start pre-processing text information with natural language tools. Some exploratory data analysis and visualizations end with this portion.

Before we move to the feature selection and training level, we use the NLP tools to pre-process the dataset. To divide the posts into individual tokens, we use tokeniza-tion first. Next, we remove all URLs, punctuations, and stop words that might lead to erroneous results if ignored. Then, we apply stemming to reduce the words to their root form and group related words together.

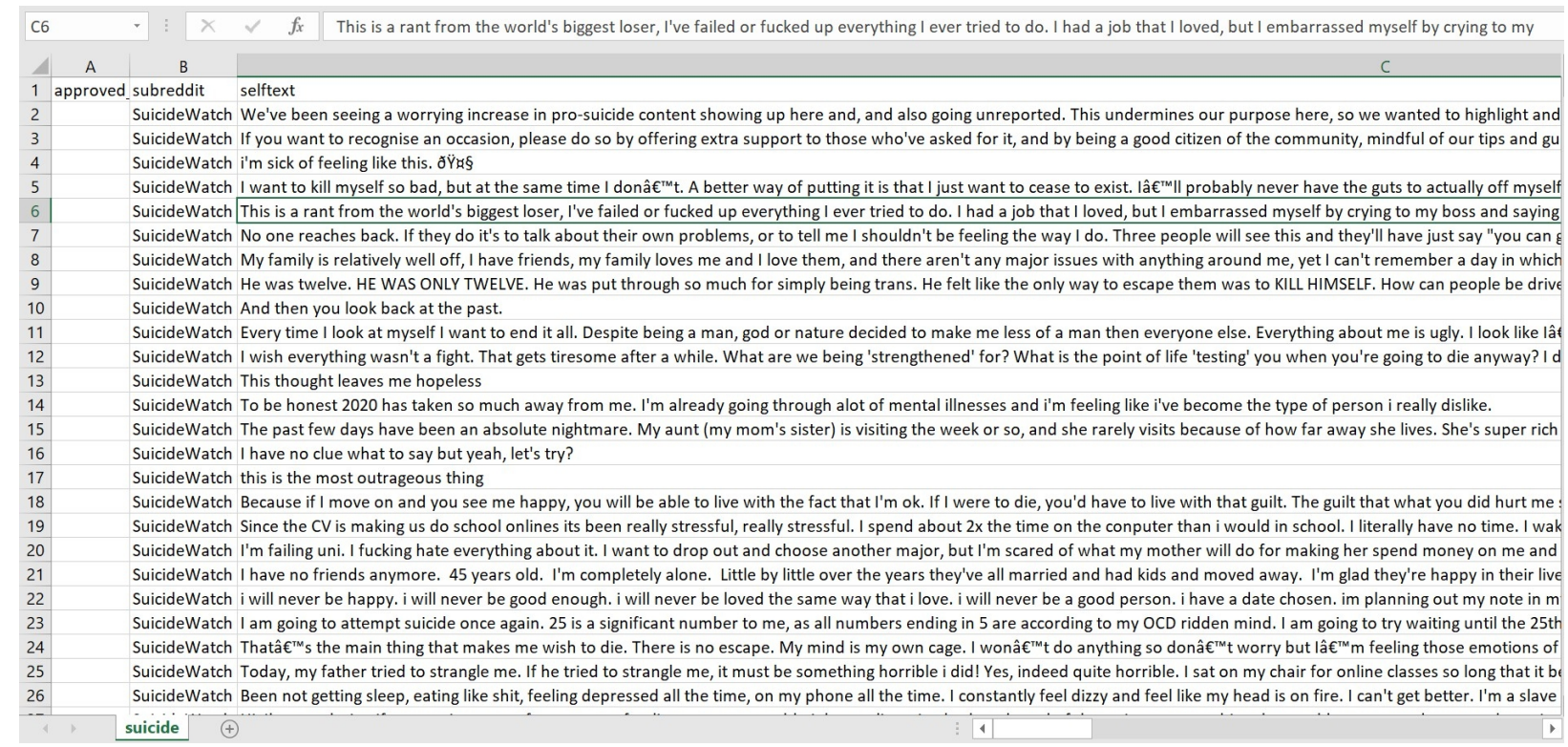

\section{Figure 1: Dataset}

Extracting functionality: After data pre-processing, we feed our models with the characteristics which reflect the language habits of users in Reddit forums. To ex-plore the linguistic use of the users in the blogs, we use the LIWC dictionary, LDA topics, and N-gram features. To encode words to be used by different classifiers, these methods of text encoding are used. N-gram modelling is used to analyse the characteristics of the documents. In text mining and NLP, it is widely used to calcu-late the probability of co-occurrence of each input sentence as a unigram and bigram as a function for depression detection [9],[40]. As a numerical statistic for n-gram modelling, we use the Term Frequency-Inverse Document Frequency (TF-IDF), where the value of a word is highlighted with respect to each 
corporate document.

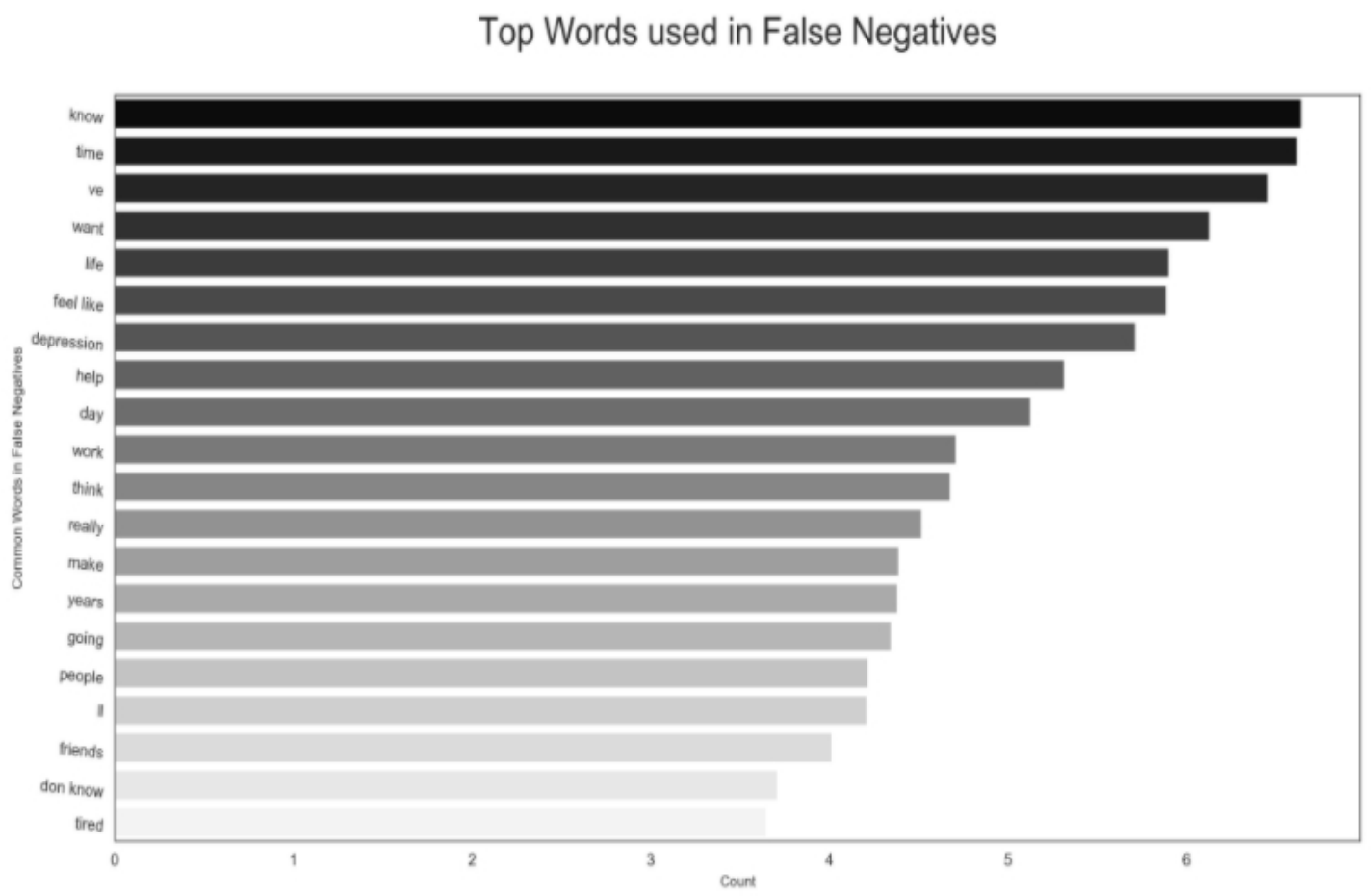

Figure 2: Bar Plot for Top Words

\section{Proposed Methodology Architecture}

In computational linguistics, topic modelling is an important method to reduce the input of textual data feature space to a fixed number of topics [20]. Hidden topics such as subjects connected with anxiety and depression can be extracted from the selected documents via the unsupervised text mining approach. It is not generated by a predetermined collection of pre-established terms in contrast to LIWC. However, it produces a category of non-labelled terms automatically. 


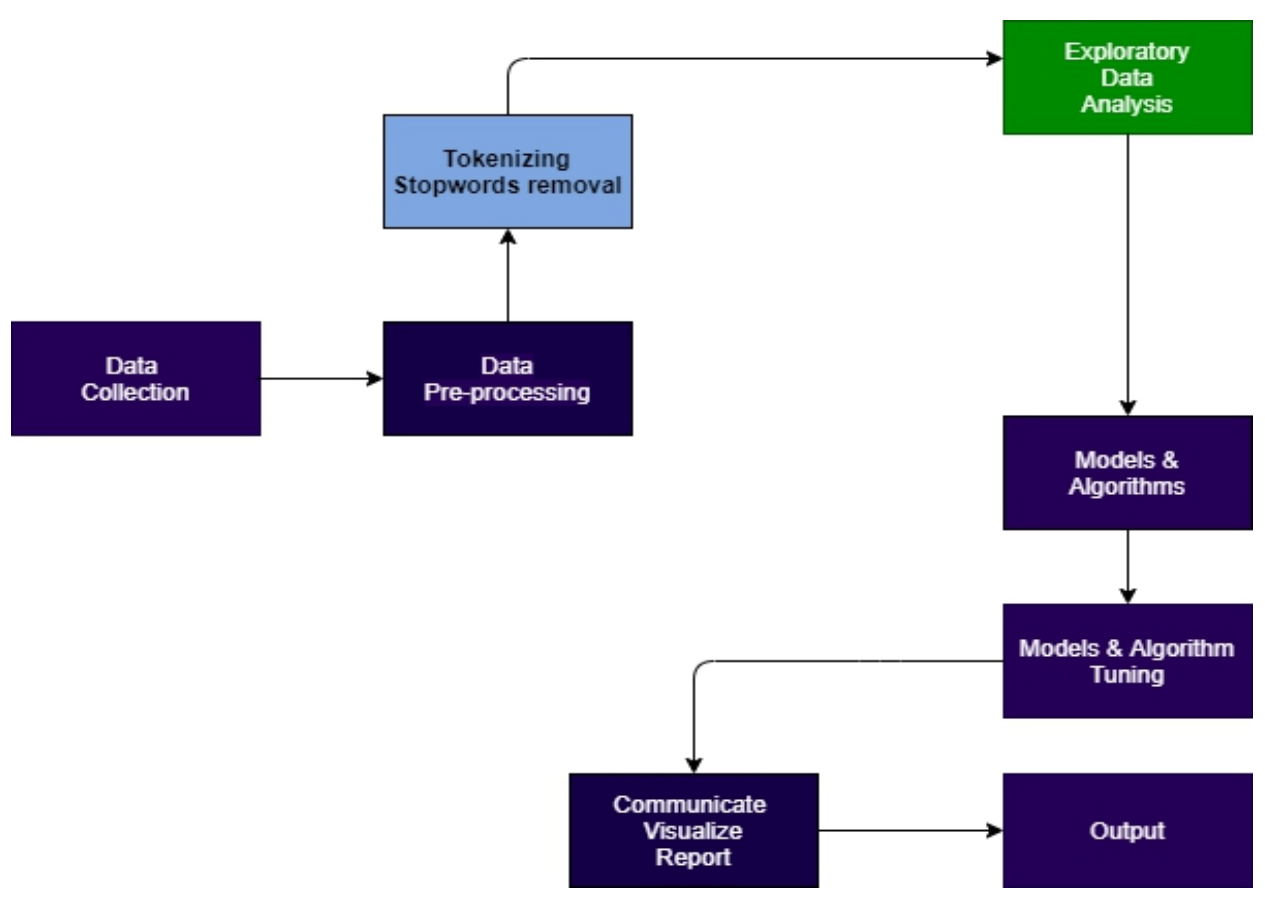

Figure 3: Block Diagram of Proposed System

We use classifying methods to quantify the probability of depression among the users in order to quantify the presence of depression. Using Logistic Regression, Support Vector Machine, Random Forest, Adaptive Boosting and Multilayer Perceptron classifier, the pro-posed structure is built.

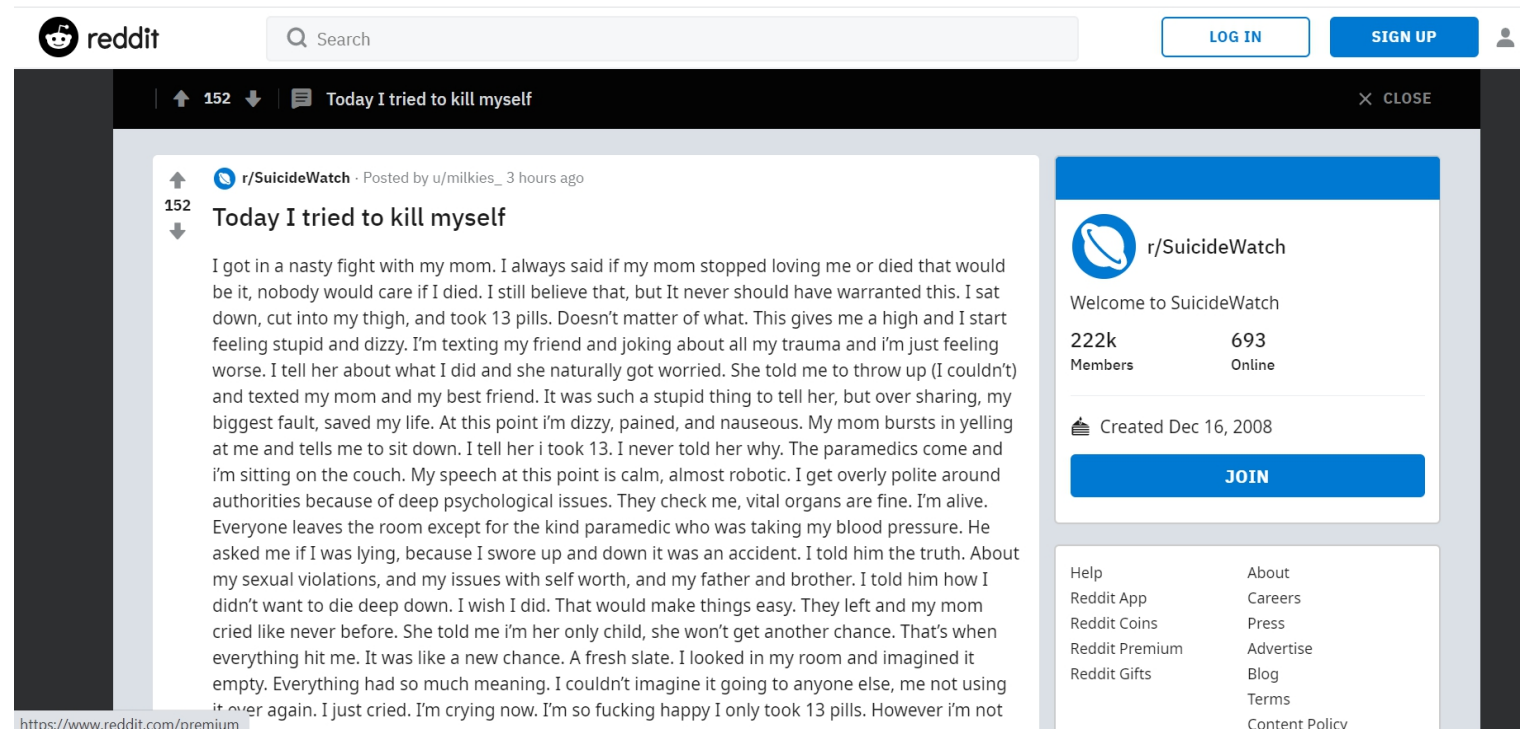

Figure 4: Live Blog Data

Adaptive Boosting (AdaBoost) is an ensemble technique that can make one strong classifier combine several weak classifiers[56]. It is commonly used for problems of binary classifica-tion, A special case of the artificial neural network, Multilayer Perceptron ( MLP) is mostly used for modelling complex relationships between the input and output layers[58]. It is able to discern data that is not only non-linearly separable due to its many layers and non-linear activation[59]. In our analysis, we used the MLP method and two hidden layers with 4 and 16 perceptrons to correct all the characteristics in 
order to ensure accuracy of the compari-son.

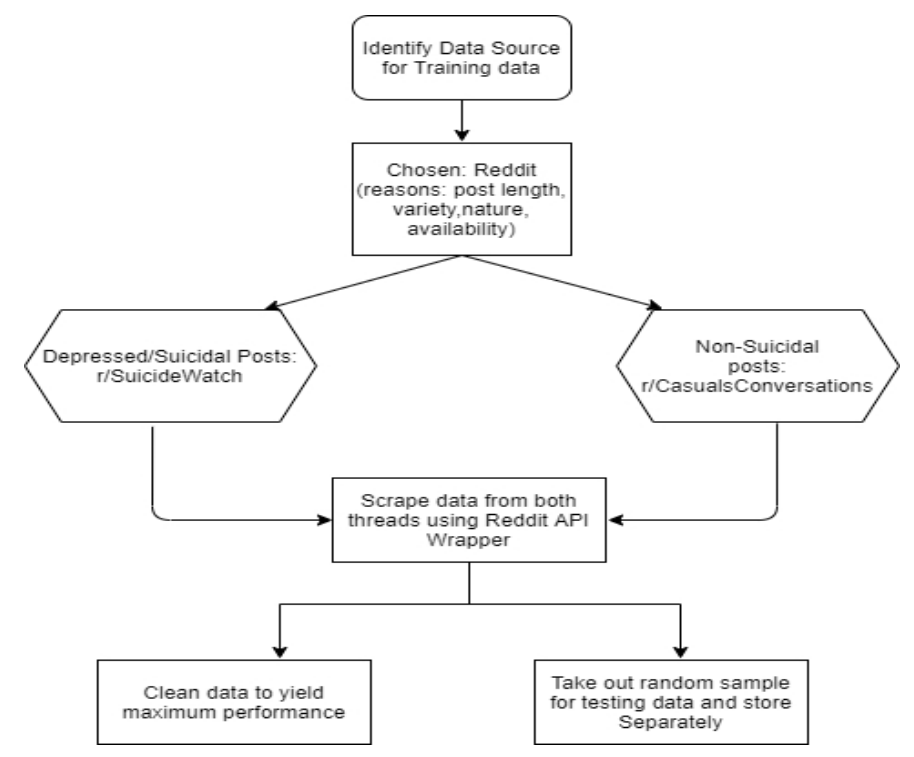

Figure 5: Flow of Proposed System

Since depression also affects psychomotor functions[60], we can find terms that represent the symptoms of low energy, exhaustion or inverse insomnia and hyperactivity (tired, I 'm tired or sleepy). It is also articulated somatically (my brain, discomfort, hurt) via the symp-toms of the body. Unigrams and bigrams in regular posts, unlike depression-indicative posts, contain the terms identifying the events that happened quite in the past (time, month ago, year ago, last year).

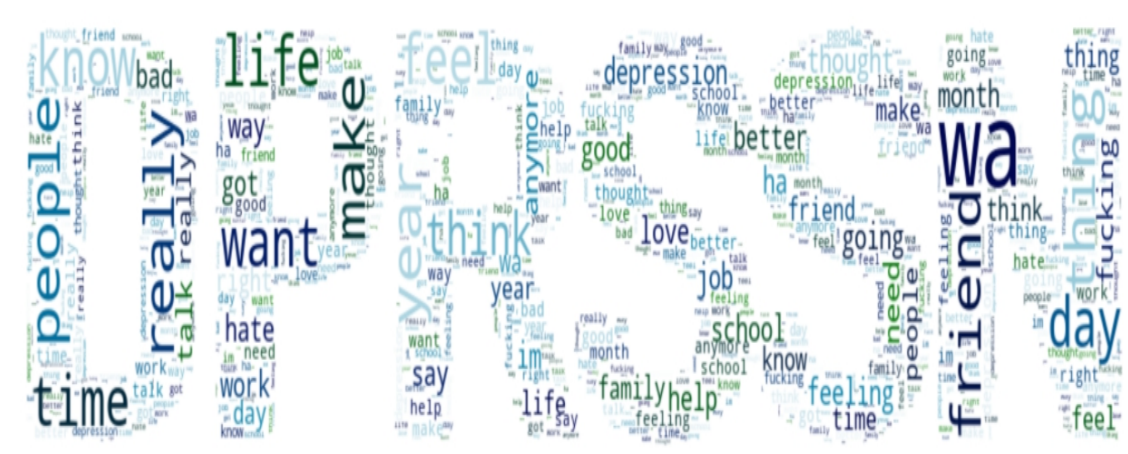

Figure 6: Most Top Words Used

To evaluate the connexion between the textual data and the features themselves, we select-ed 68 out of 95 characteristics. In view of psycholinguistic characteristics resulting in asso-ciation provided in the features extraction, we transformed every depressive and non-depressive post into numerical values. The Psychological Mechanisms (0.19) followed by the Linguistic Aspects (0.17) and Personal Concerns (0.16) show the greatest correlation. c)GSM Module Working. 


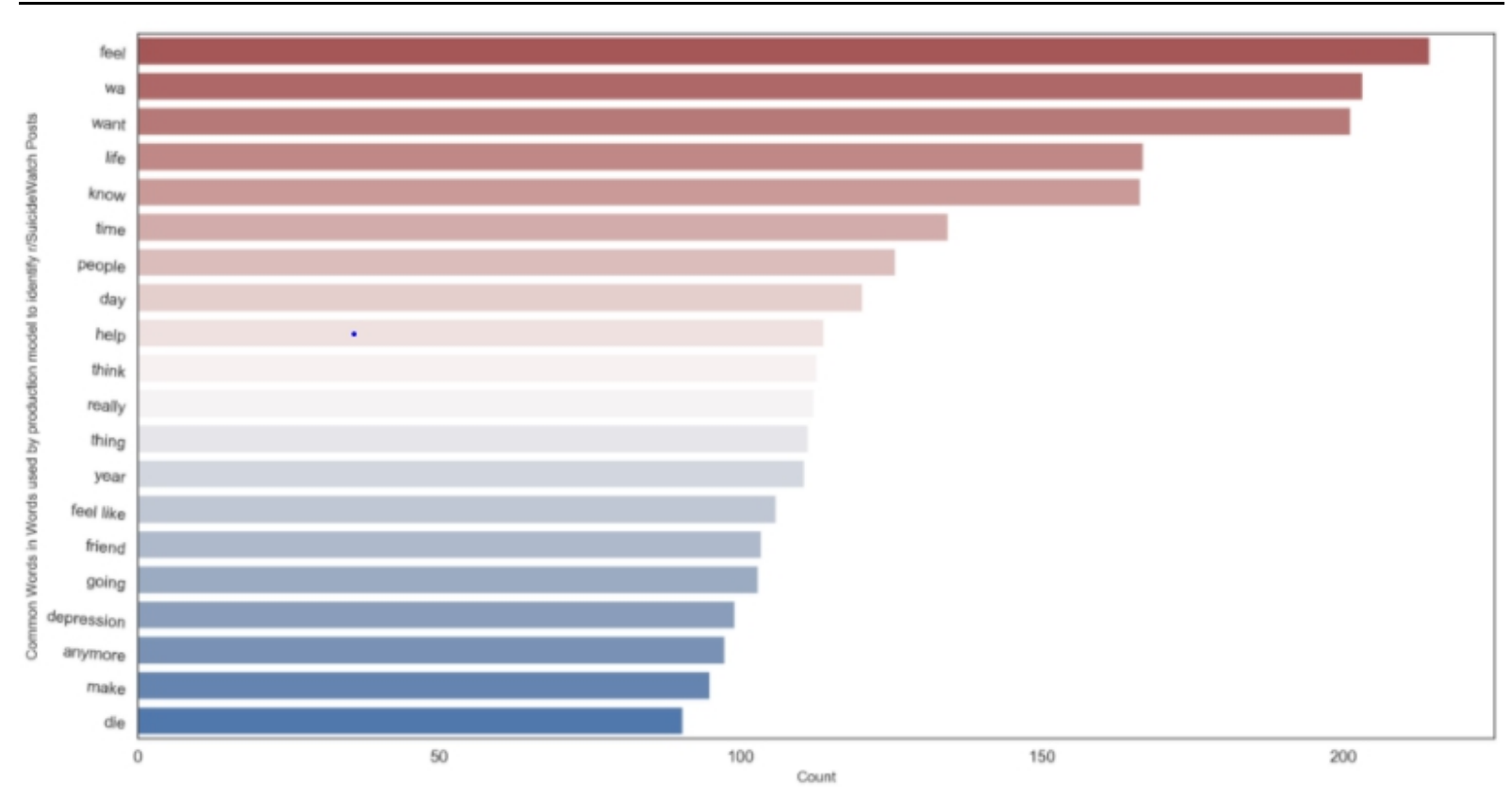

Figure 7: Occurrence of Top Positive Words Used

The findings indicate that depressed individuals use more self-oriented references with respect to the mental concentration of depressed and non-depressed users and prefer to shift their attention to themselves (I, me, and mine) (0.17). The work of [13], [14] is confirmed by the findings. With a

stronger focus on the present and future, their posts contain more negative feelings, depression and anxiety. Based on our results, when applied to the design tools, LIWC may play an effective role in data detection models.

\section{Word Comparison}

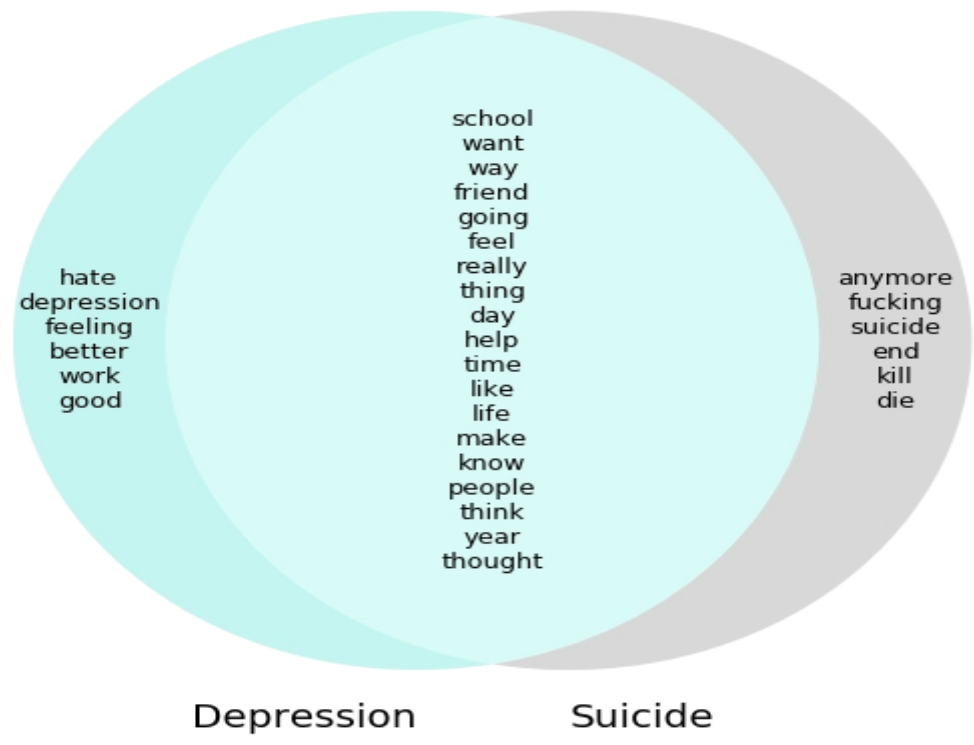

Figure 8: Word of Comparison.

We developed a topic model to quantify the hidden topics extracted from the posts, which acts as a depression triggering point. LDA requires that the number of topics produced be specified. Any parameter change can trigger a change in the accuracy of the classification. For this purpose, an 
acceptable value needs to be identified.

\section{Results}

Deployment done using flask where as in user right in text box it will call the backend API and through that will give the result as shown in fig. 9. There is an $90 \%$ accuracy of prediction depression text data. The data was extracted from Reddit and categorised by subreddit, with VADER Sentiment analysis performed on each request. Separately from the test set, a training set of data was developed. The training set of data was pruned to exclude submissions that were overly positive for $\mathrm{r} /$ SuicideWatch and excessively negative for $\mathrm{r} /$ CasualConversation. This was achieved to increase the training data's divergence. The data was fitted and trans-formed using a Spark Machine Learning Pipeline that generated features based on TF-IDF analysis and the negative sentiment.

\section{ALGORITHMS}

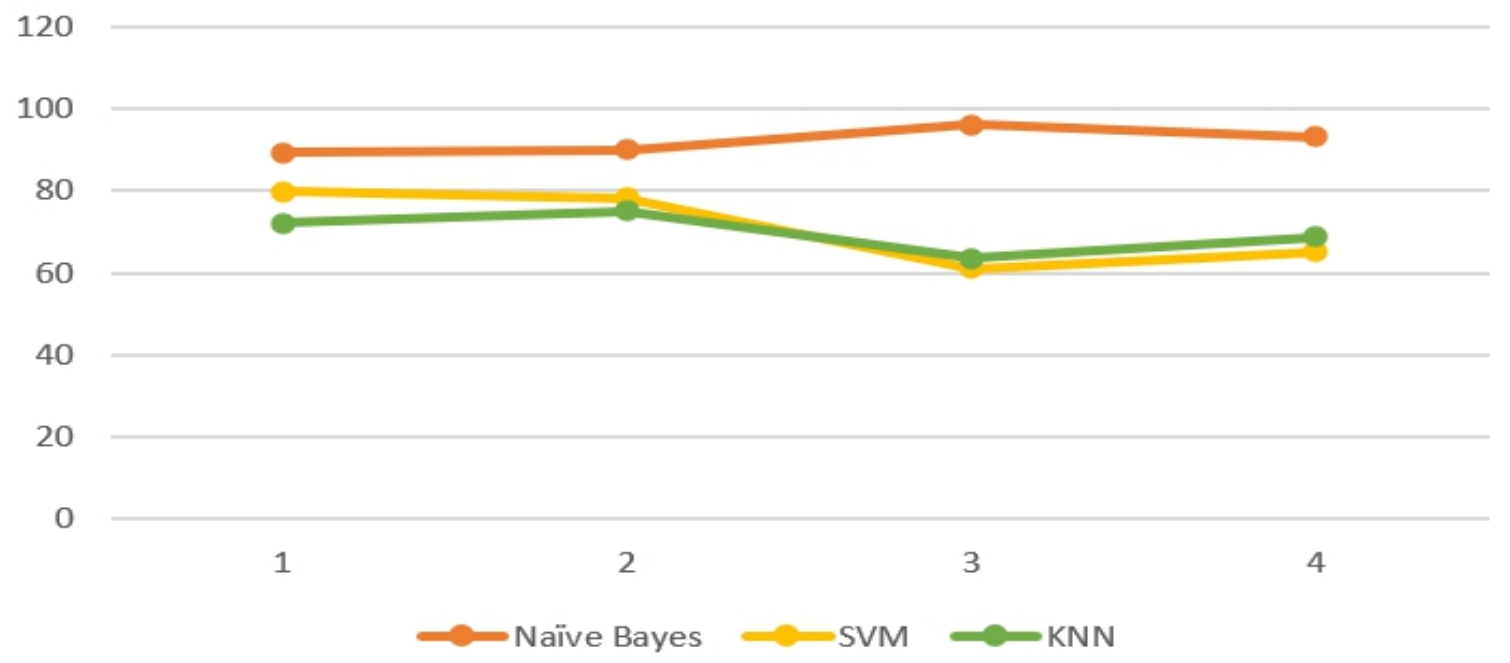

Figure 8: Algorthm

Comparative Analysis with Paper Title Sentiment Analysis from Bengali Depression dataset using Machine Learning they took dataset in Bengali Language which was static it was been collected from different places and after that applied Machine Leaning algorithm where else in Prediction of depression using Machine Leaning and NLP approach used the live dataset of Reddit.com and used 10 $\mathrm{k}$ Fold validation which gave better Accuracy compared to existing paper [14].

Table 1: Result of Algorithm

\begin{tabular}{|l|l|l|l|l|l|}
\hline Model & Accuracy & Precision & Recall & F1 Score & Model \\
\hline $\begin{array}{l}\text { Naïve } \\
\text { Bayes }\end{array}$ & 89.36 & 89.90 & 96.00 & 93.00 & $\begin{array}{l}\text { Naïve } \\
\text { Bayes }\end{array}$ \\
\hline SVM & 79.70 & 78.25 & 78.99 & 82.10 & SVM \\
\hline KNN & 72.10 & 75.00 & 63.40 & 68.70 & KNN \\
\hline
\end{tabular}


International Journal of Intelligent Communication, Computing and Networks

Open Access Journal (ISSN: 2582-7707)

https://doi.org/10.51735/ijiccn/001/16

Comparative Analysis with Paper Depression detection using Emotion Artificial Intelligence after comparing with the algorithms it can be seen that Naïve Bayes Algorithm has got good accuracy of $89.36 \%$ in this used live Reddit.com dataset of blog and applied $10 \mathrm{k}$ Fold Validation [15].

\section{Discussion}

VADER Sentiment analysis, and was vectorized after the pruning process was com-pleted. A Naive Bayes multinomial classifier and a KNN model are trained using the newly transformed results. This model was used to predict whether a particular test set submission will be posted in $\mathrm{r} /$ SuicideWatch or $\mathrm{r} /$ CasualConversation. The accu-racy was estimated to be $90 \%$ plus or minus $2 \%$.

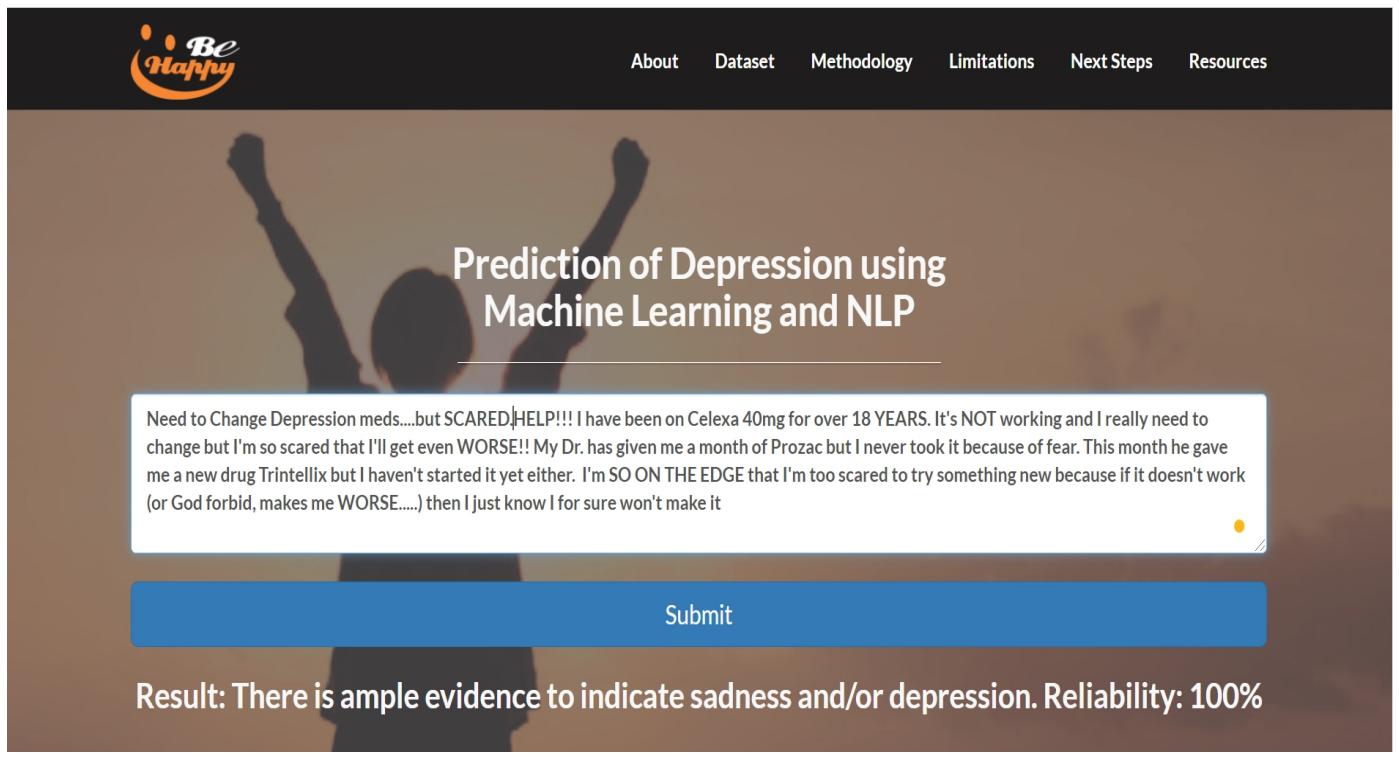

Figure 9: Deployment

\section{Conclusion}

The detection of mental health issues is possible at early stage. Analysis done by the model helps to detect issues precisely and accurately that will give better output than the human analysis. Since the prediction model uses a greater number of records, performing analysis will yield accurate results. Through this project learned Wrap Scrapping of Live data using Reddit.com and Model Training and Lastly Deployment of Project using Flask.Basically, Naïve Bayes, SVM and KNN classification will be used to final classify the text used. And finally achieving the outcome was suicidal note or not as the post. After model prediction deployment, it is possible to allow users to input text and receive predictions about their mental state. prediction result is $90 \%$.

\section{Acknowledgments}

I would like to take this opportunity to thank Dr. R.R. Sedamkar, Professor in the COMP Department and Director of the IQAC at TCET, for his unwavering support and encouragement during my P.G. study. This would not have been possible without his kind encouragement and assistance. I appreciate his prompt input, which allowed me to effectively track and schedule the process I'd like to express my gratitude to Dr. R.R. Sedamkar for his support and encouragement during my Post Graduation. I would also thank External 
International Journal of Intelligent Communication, Computing and Networks

Open Access Journal (ISSN: 2582-7707)

https://doi.org/10.51735/ijiccn/001/16

Mentor Kirti Sharma, Data Scientist in U.S

I'd also like to express my gratitude to Dr. B. K. Mishra, Principal, Thakur College of Engineering and Technology, for his support and for providing an excellent learning atmosphere as well as adequate facilities. I am grateful to all of my M.E. teachers for their helpful advice and encouragement. I'd like to express my gratitude to all of the faculty members, as well as non-teaching staff and colleagues, for their assistance. Last but not least, I am grateful to all of my family members for their unwavering support and encouragement during the project.

\section{References}

1. D. Greene and P. Cunningham. "Practical Problem of Diagonal Dominance in Kernel Document Clustering", Proc. ICML 2006.

2. J. Grimmer and B. M. Stewart, "Text as Data: The Promise and Pitfalls of Au-tomatic Content Analysis Methods for Political Texts,” Political Analysis, Janu-ary 2013, pp. 1-31, doi:10.1093/pan/mps028.

3. F."Machine Learning in Automated Text Categorization,” ACM Computing Surveys, vol. 34, No. 1, March 2002, pp. 1-47.

4. J. Grimmer and B. M. Stewart, "Text as Data: The Promise and Pitfalls of Au-tomatic Content Analysis Methods for Political Texts," Political Analysis, Janu-ary 2013, pp. 1-31, doi:10.1093/pan/mps028.

5. G. D. Guo, H. Wang, D. Bell, Y. X. Bi and K.Greer, "Using kNN model for au-tomatic text categorization," Soft Computing, 10(5), pp. 423- 430, 2006.

6. A. S. Patil, B.V. Pawar, "Automated Classification of Web Sites using Naive Bayesian Algorithm," Proceedings of the International MultiConference of En-gineers and Computer Scientists, Hong Kong 2012, Vol. I, 14-16.

7. L. Jiang, C. Li, S. Wang, and L. Zhang, "Deep feature weighting for naïve Bayes and its application to text classification,” Engineering Applications of Ar-tificial Intelligence, vol. 52, June 2016, pp. 26-39. Lasisi and A A. Ajisafe, "Development of stripe biometric based Fingerprint Authentications Systems in Automated Teller Machines,” 2012, IEEE, ISBN. 978-1 -4673-2488-5, pp. 1 72- 175.

8. Hamid Haqani, Mir Saleem, Shoaib Amin Banday, Ab RoufKhan, "Biometric verified Access Control of Critical Data on a Cloud," International Conference on Communication and Signal Processing, April 3-5, 2014,India.

9. Q. Yuan, G. Cong, and N. M. Thalmann, "Enhancing Naive Bayes with Various Smoothing Methods for Short Text Classification,"WWW 2012 Companion, April 16-20, 2012, Lyon, France, ACM 978$1-4503-1230-1 / 12 / 04$.

10. V. Lertnattee and T. Theeramunkongt, "Analysis of Inverse Class Frequency in Centroid-based Text Classification," International Symposium on Communica-tion and Information Technologies 2004 (ISCIT 2014), pp. Japan, October 26-29, 2004.2014 | London,UK.

11. "Evaluation: From Precision, Recall, and F-Factor to ROC, Informedness, Markedness, and Correlation," by David M W Powers. Flinders University of South Australia, Technical Report SIE-07-001, December 2007.

12. Abdul Razaque, Fathi H. Amsaad, Chaitanya Kumar Nerella, Musbah Abdulga-der, Harsha Saranu, 
“Multi-Biometric System Using Fuzzy Vault”, 978-1-4673-9985-2/16/\$31.00@2016 IEEE

13. Debanjan Sadhya, Bodhi Chakraborty, "Review of key-binding-based biometric protection schemes", IET Biom. (C) The Institution of Engineering and Technology2016

14. Xin $\mathrm{Xu}$ "An Online Biometric Identification System Based on Two Dimen-sional Fisher Linear Discriminant”, 978-1-4673- 9098- 9/15/\$3.00 @2015IEEE.

15. Latha Parthiban "Trusted framework for online cloud using multi-factor authentication protection gateway", Parthiban Journal of Cloud Computing: Advances, Systems and Applications (2015)4:22.

16. Dilsizian SE, Siegel EL. Artificial intelligence in medicine \& cardiac imaging: harnessing big data 2014;16(1):1-8.

17. Markonis D, Schaer R. Using MapReduce for large-scale medical image analysis. In: 2012 IEEE Second International Conference on Healthcare Informatics, La Jolla, California; IEEE. 2012:1.

18. Shortliffe, Cimino JJ. Biomedical Informatic Springer; 2014.

19. Hay SI, George DB, Moyes CL. Big data opportunities for global infec-tious disease surveillance. PLoS Med. 2013;10(4): e101413.

20. Francis J, Kerr E, Advancing evidence-based care for dia-betes: lessons from the Veterans Health Administration J. Health Affairs. 2007; 26(2): w156-68. 